\title{
Influence des bactéries psychrotrophes sur les qualités organoleptiques de fromages à pâte molle
}

\author{
par \\ J. P. DUMONT*, G. DELESPAUL**, B. MIGUOT** \\ et J. ADDA*
}

\section{INTRODUCTION}

La généralisation de la collecte de laits refroidis et l'utilisation de plus en plus fréquente de laits de report de mauvaise qualité bactériologique n'a pas été sans poser de graves problèmes à l'industrie laitière.

Une des conséquences de la conservation du lait à basse température est le développement d'une flore psychrotrophe susceptible non seulement de modifier l'aptitude du lait à la coagulation et de rendre plus difficile la croissance des levains lactiques mais aussi de provoquer des défauts organoleptiques majeurs en raison de la production d'enzymes lipolytiques et protéolytiques qui pour certaines d'entre elles résistent à la pasteurisation.

La responsabilité de ces bactéries dans l'apparition de défauts de goût a été démontrée dans le cas du lait, du beurre et de la crème. Par contre, l'incidence de la contamination du lait par des bactéries psychrotrophes sur les qualités organoleptiques des fromages n'a été que peu étudiée.

C'est pourquoi il a semblé intéressant d'étudier sur des fabrications de pâtes molles l'effet du report du lait à basse température pendant un temps variable sur la qualité des fromages et les composants volatils responsables de l'arôme.

\footnotetext{
* Laboratoire de Technologie Laitière, I.N.R.A. - 78350 Jouy-en-Josas.

** Centre de Recherche des Fromageries Bel - 41100 Vendôme.

Ce travail a bénéficié du concours financier de la D.G.R.S.T. (contrat $\mathrm{n}^{\circ} 7570574$ ).
} 


\section{MATERIEL ET METHODES}

\section{II.1. Echantillons}

Les essais de fromagerie ont été réalisés en utilisant des laits de grand mélange présentant initialement une bonne qualité bactériologique et conservés à l'état cru à $+5^{\circ} \mathrm{C}$ de façon à laisser une flore psychrotrophe plus ou moins importante se développer.

Après des temps de report de $2 \mathrm{j}, 6 \mathrm{j}$ et $9 \mathrm{j}$, le lait a été pasteurisé à $72^{\circ} \mathrm{C}$ et on a réalisé des fabrications de pâtes molles du type Camembert qui ont été affinées durant $35 \mathrm{j}$. Les fromages ont alors été soumis à un jury de dégustation qui a noté leur texture et leur goût. Les échantillons destinés aux analyses ont été immédiatement congelés et conservés à $-30^{\circ} \mathrm{C}$.

Différents dosages ont été effectués sur ces fromages :

- extrait sec, teneur en matière grasse, rapports gras/sec et eau/ESD en sortie de saumure et en fin d'affinage ;

- $\mathrm{pH}$ et teneur en $\mathrm{NaCl}$ à la sortie de saumure ;

- azote total et azote soluble à l'alun à l'issue de l'affinage.

\section{II.2. Analyses}

\section{II.2.1. Analyses bactériologiques}

La flore totale et la flore psychrotrophe ont été dénombrées sur milieu PCA après incubation à $30^{\circ} \mathrm{C}$ pendant $3 \mathrm{j}$ et à $7^{\circ} \mathrm{C}$ pendant $10 \mathrm{j}$ respectivement.

\section{II.2.2. Mesure de la lipolyse}

La matière grasse est obtenue par centrifugation du fromage (centrifugeuse Servall SS34, $32000 \mathrm{~g}, 30 \mathrm{mn}$ ). On dissout $1 \mathrm{~g}$ de matière grasse dans $10 \mathrm{ml}$ d'un mélange éthanol : éther éthylique $1: 1$ et on titre son acidité libre avec de la potasse alcoolique $0,01 \mathrm{~N}$ en présence de phénolphtaléine. Les acides gras libres ont été dosés par une méthode colorimétrique (Lauwerys, 1969) à partir d'une suspension de fromage dans l'eau obtenue à l'aide d'un Ultraturrax.

\section{II.2.3. Dosage des composés monocarbonylés totaux}

Les composés monocarbonylés totaux ont été isolés et dosés sous forme de 2,4-dinitrophénylhydrazones (Schwartz et Parks, 1963 ; Schwartz et al., 1963).

\section{II.2.4. Extraction des composés volatils}

Les composés volatils ont été extraits par une méthode de distillation sous vide poussé (Dumont et al., 1974). A l'issue de la distillation le contenu des pièges est amené à $\mathrm{pH}$ 9,0 par addition de soude normale et les composés neutres sont extraits par du Fréon 11, 
puis par du dichlorométhane. Ces extraits ont été réduits à un volume de quelques $\mathrm{ml}$ par distillation sous une colonne adiabatique remplie d'anneaux de Raschig en verre, puis concentrés jusqu'à un volume de $200 \mu \mathrm{l}$ en utilisant une courte colonne de Dufton à spires de nickel. Les extraits Fréon et dichlorométhane ainsi obtenus ont été séparés par chromatographie en phase gazeuse en utilisant un appareil Girdel 3000 équipé d'une colonne capillaire $150 \mathrm{~m}$ en acier inoxydable (d.i. $0,75 \mathrm{~mm}$ ) revêtue de SF 96 - Igepal CO 880 95:5 couplée à un spectromètre de masse AEI MS 20 organique par un séparateur à membrane. Les analyses sont effectuées en utilisant une programmation de température entre $30^{\circ} \mathrm{C}$ et $130^{\circ} \mathrm{C}$ à raison de $1,50 / \mathrm{mn}$ après une période isotherme à $30^{\circ} \mathrm{C}$.

Les composés volatils contenus dans les distillats aqueux correspondant à $50 \mathrm{~g}$ de fromage ont été étudiés par une méthode de Head Space à partir d'un volume de $500 \mathrm{ml}$ (Von Sydow et al., 1970) et en utilisant une colonne capillaire en verre de $80 \mathrm{~m}$ (d.i. 0,75 mm) de même nature que celle utilisée en couplage. Les composés soufrés ont été étudiés de manière analogue (Qvist et Von Sydow, 1974).

\section{RESULTATS}

\section{III.1. Analyse du lait cru}

Le tableau 1 donne les résultats des dénombrements effectués sur le lait cru après conservation pendant $2 \mathrm{j}, 6 \mathrm{j}$ et $9 \mathrm{j}$ à $+5^{\circ} \mathrm{C}$. On peut voir que la flore psychrotrophe devient rapidement dominante.

\section{III.2. Analyse des fromages}

Nous avons rassemblé dans les tableaux 2 et 3 les résultats analytiques concernant les fromages en sortie de saumure et après

TABLEAU 1. - Analyse du lait cru

\begin{tabular}{|c|c|c|c|}
\hline & Coliformes & Germes totaux & $\begin{array}{c}\text { Germes } \\
\text { psychrotrophes }\end{array}$ \\
\hline $48 \mathrm{~h}$ & 570 & $63 \times 10^{3}$ & $12 \times 10^{2}$ \\
\hline $6 j$ & $13 \times 10^{3}$ & $91 \times 10^{4}$ & $94 \times 10^{4}$ \\
\hline $9 \mathrm{j}$ & $29 \times 10^{4}$ & $63 \times 10^{6}$ & $44 \times 10^{6}$ \\
\hline
\end{tabular}


$T A B L E A U 2$

Analyse des fromages sortie saumure

\begin{tabular}{l|c|c|c|c|c|c}
\hline & ES & MG & G/S & Eau/ESD & pH & NaCl \\
\hline & & & & & & \\
\hline & & & & & \\
$48 \mathrm{~h}$ & 44,83 & 21,50 & 47,96 & 2,36 & 4,45 & - \\
$6 \mathrm{j}$ & 44,83 & 21,37 & 47,68 & 2,35 & 4,63 & 2,02 \\
$9 \mathrm{j}$ & 45,83 & 22,12 & 48,28 & 2,28 & 4,60 & 1,87 \\
\hline
\end{tabular}

TABLEAU 3

Analyse des fromages à $35 \mathrm{j}$

\begin{tabular}{l|c|c|c|c|c|c|c|c}
\hline & ES & MG & pH & G/S & $\begin{array}{c}\text { Eau/ } \\
\text { ESD }\end{array}$ & $\begin{array}{c}\mathbf{N} \\
\text { total }\end{array}$ & $\begin{array}{c}\mathbf{N} \\
\text { soluble }\end{array}$ & $\begin{array}{c}\text { p. 100 } \\
\text { affin. }\end{array}$ \\
\hline & & & & & & & & \\
\hline $48 \mathrm{~h}$ & 49,46 & 24,75 & 6,92 & 50,04 & 2,04 & 3,33 & 0,72 & 80 \\
$6 \mathbf{j}$ & 46,85 & 23,25 & 6,74 & 49,63 & 2,25 & 3,23 & 0,75 & 70 \\
$9 \mathbf{j}$ & 48,46 & 24,50 & 6,51 & 50,56 & 2,15 & 3,24 & 0,77 & 40 \\
\hline
\end{tabular}

affinage. On remarquera que la conservation du lait à basse température entraîne une diminution notable du pourcentage d'affinage (défini comme le rapport de la surface de la zone apparaissant affinée à la surface totale de la coupe).

\section{III.3. Notes de dégustation (tab. 4)}

Les notes globales de texture et de goût baissent avec l'allongement de la conservation au froid. La texture est plus compacte et moins onctueuse dans les fromages fabriqués avec du lait gardé longtemps au froid. Ces fromages sont plus amers et deviennent très rances. On notera l'absence de caractère ammoniacal.

III.4. L'examen des profils chromatographiques montre une évolution très nette (fig. 1). On peut tout d'abord constater une augmentation 


\section{TABLEAU 4}

Examen organoleptique à $35 \mathrm{j}$

\begin{tabular}{|c|c|c|c|c|}
\hline & & $48 \mathrm{~h}$ & $6 \mathrm{j}$ & $9 \mathrm{j}$ \\
\hline \multirow{3}{*}{ Texture } & Note globale/5 & 3,50 & 3,25 & 3,00 \\
\hline & $\begin{array}{l}\text { Appréciations : } \\
\text { onctueuse } \\
\text { fondante } \\
\text { plâtreuse } \\
\text { collante } \\
\text { granuleuse } \\
\text { compacte }\end{array}$ & $\begin{array}{l}\text { M } \\
\text { M } \\
\text { A } \\
\text { f } \\
\text { A } \\
\text { A }\end{array}$ & $\begin{array}{l}\mathrm{f} \\
\mathrm{M} \\
\mathrm{A} \\
\mathrm{f} \\
\mathrm{s} \\
\mathrm{f}\end{array}$ & $\begin{array}{l}\text { A } \\
\mathbf{f} \\
\mathrm{f} \\
\mathrm{f} \\
\mathrm{f} \\
\mathrm{M}\end{array}$ \\
\hline & Note globale/5 & 2,50 & 1,83 & 1,70 \\
\hline Goût & $\begin{array}{l}\text { Appréciations : } \\
\text { amer } \\
\text { piquant } \\
\text { moisi } \\
\text { rance } \\
\text { ammoniacal } \\
\text { acide }\end{array}$ & $\begin{array}{l}\text { M } \\
\mathrm{f} \\
\mathrm{A} \\
\mathrm{A} \\
\mathrm{f} \\
\mathrm{s}\end{array}$ & $\begin{array}{l}\text { M } \\
\text { s } \\
f \\
\text { M } \\
\text { f } \\
\text { A }\end{array}$ & $\begin{array}{l}\text { F } \\
f \\
f \\
F \\
A \\
\text { f }\end{array}$ \\
\hline
\end{tabular}

$\mathrm{A}=$ absence $; \mathrm{s}=$ seuil $; \mathrm{f}=$ faible $; \mathrm{M}=$ modéré $; \mathrm{F}=$ fort.

de la surface des pics correspondant aux méthylcétones en particulier des termes à longue chaîne saturée (2-octanone, 2-nonanone, 2-décanone, 2-undécanone, 2-dodécanone, 2-tridécanone) ou insaturés (2-nonénone, 2-undécénone, 2-tridécénone). Cette augmentation est confirmée par les dosages colorimétriques puisqu'il apparaît (tab. 5) que les valeurs passent de 2,76 à 16,52 $\mu \mathrm{m}$ pour les échantillons fabriqués avec des laits conservés respectivement 2 j et 9 j à $5^{\circ} \mathrm{C}$. Corrélativement on note une forte augmentation des alcools secondaires à

\section{$T A B L E A U 5$}

Composés monocarbonyles totaux pour $100 \mathrm{~g}$ de fromage

\begin{tabular}{ll|ll|ll}
\hline $2 \mathrm{j}$ & $2,76 \mu \mathrm{M}$ & $6 \mathrm{j}$ & $14,08 \mu \mathrm{M}$ & $9 \mathrm{j}$ & $16,52 \mu \mathrm{M}$ \\
\hline
\end{tabular}




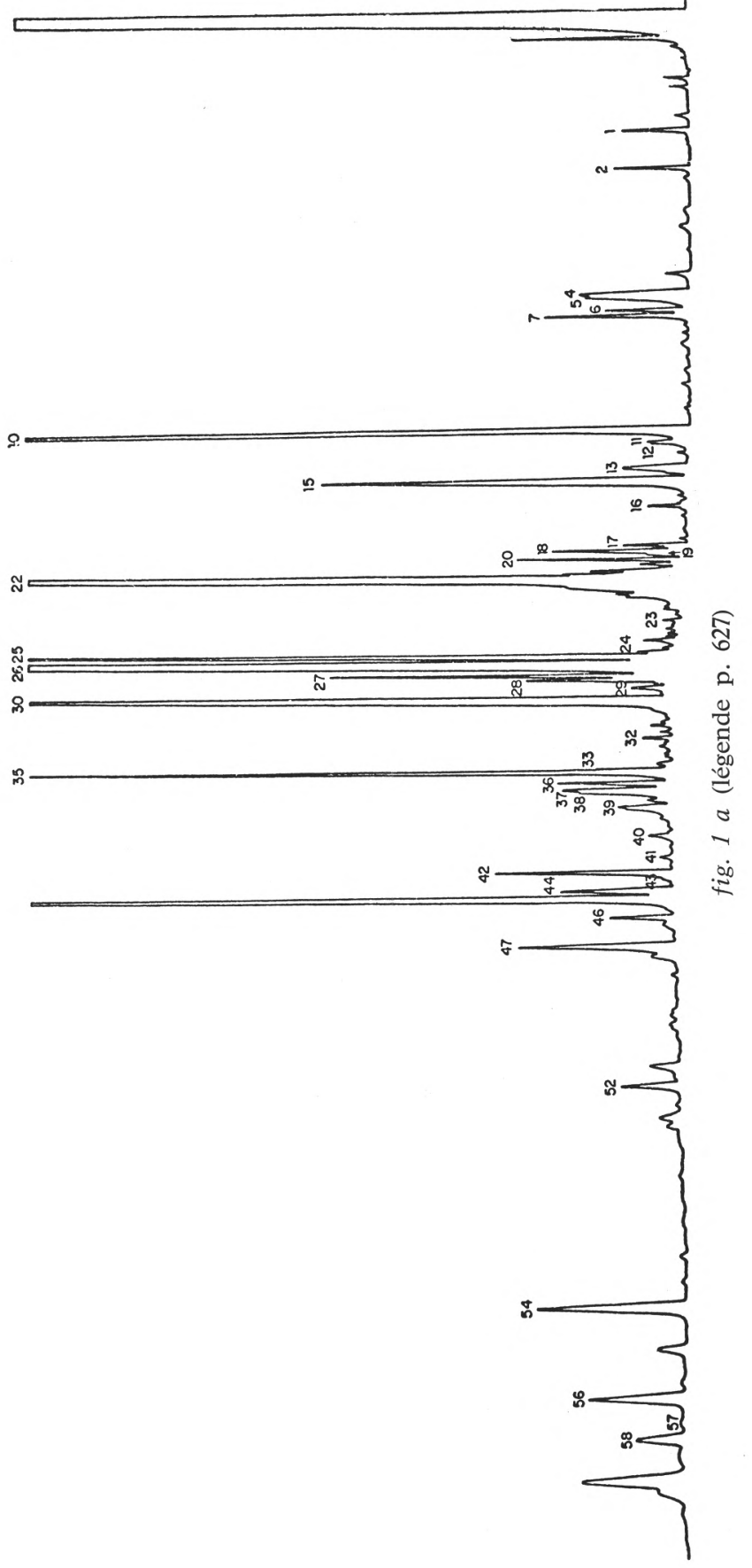




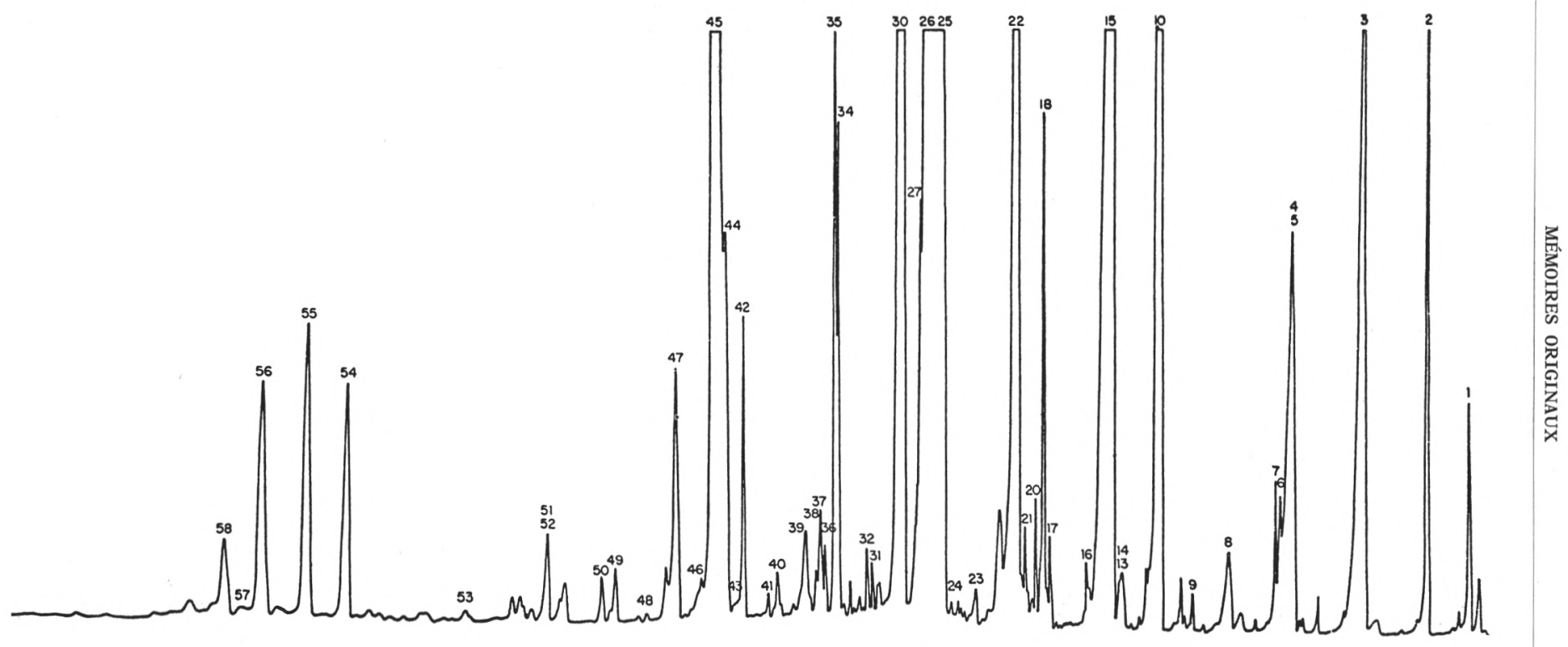

fig. 1 b (légende p. 627) 


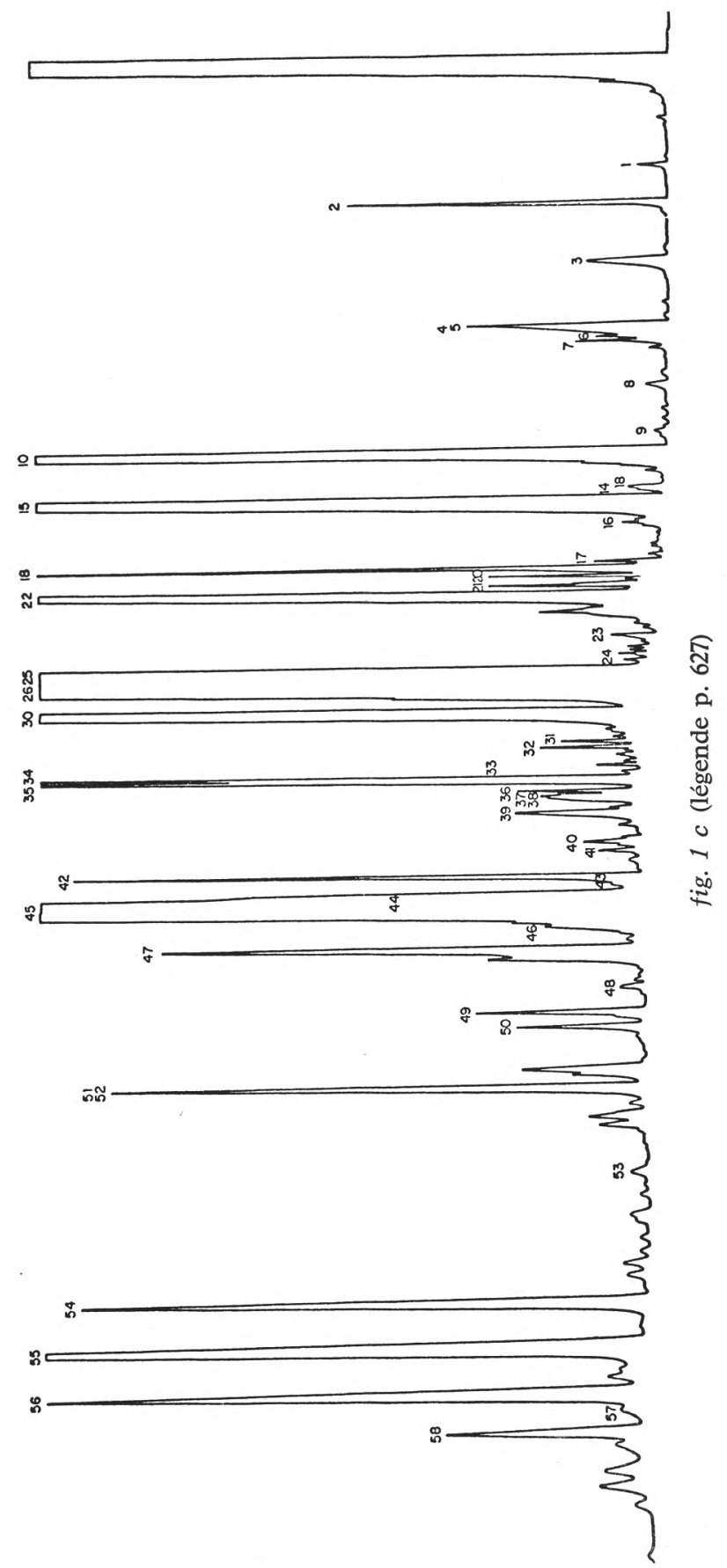


nombre impair de carbone qui est toutefois beaucoup plus importante pour le 2-heptanol que pour ses homologues supérieurs.

On notera que la 3-octanone ne subit pas pour sa part de variations notables, pas plus que les teneurs en alcools primaires et en esters.

L'octen-1-ol-3 et le 1,3-diméthoxybenzène tous deux caractéristiques des fromages à pâtes molles ne varient guère d'un échantillon à l'autre.

L'examen des profils chromatographiques obtenus par la méthode de Head Space confirme les résultats et permettent de vérifier que les teneurs élevées en 2-pentanone et 2-pentanol qui apparaissent dans l'échantillon intermédiaire ne sont pas dues à un artefact.

L'analyse des extraits dichlorométhane a permis de constater que l'on ne trouve des quantités importantes d'acétoïne que dans les fromages fabriqués avec des laits ayant subi un report court.

III.5. Les résultats de la mesure de l'acidité libre de la matière grasse (tab. 6) montrent une croissance régulière des acides gras libres à courte chaîne tandis que (tab. 7) les acides gras libres à longue chaîne suivent une évolution inverse.

\section{III.6 Analyse des produits soufrés}

Les composés soufrés sont peu abondants dans les fromages étudiés ce qui est inhabituel pour des pâtes molles. Seuls ont été décelés l'hydrogène sulfuré, le sulfure et le disulfure de méthyle qui sont présents en quantités encore plus faibles dans les échantillons fabriqués avec le lait le plus âgé.

\section{fig. 1}

Profils chromatographiques des produits volatils neutres des fromages fabriqués avec des laits conservés pendant (a) $2 \mathrm{j}$, (b) $6 \mathrm{j}$, (c) $9 \mathrm{j}$

\section{Extraits Fréon}

1. 3-méthyl-1-butanal, 2. 2-pentanone, 3. 2-pentanol, 4. 3-méthyl-1-butanol, 5. 2-hexanone, 6. hexanal, 7. éthylbutanoate, 8. 2-hexanol, 9. xylène, 10. 2-heptanone, 11. styrène, 12. heptanal, 13. hexanol, 14. méthylhexanoate, 15. 2-heptanol, 16. spectre identique à celui de la 3-octanone, 17. 3-octanone, 18. 2-octanone, 19. benzaldéhyde, 20. éthylhexanoate, 21 . décane, 22. octen-1-ol-3, 23. isoamylbutanoate, 24. phényl acétaldéhyde, 25. 2-nonénone, 26. 2-nonanone, 27. nonanal, 28. undécane, 29. octanol, 30. 2-nonanol, 31. méthylcétone, 32. méthylcétone. 33. 2-décanone, 34. éthyloctanoate, 35, 1-3-diméthoxybenzène, 36. dodécane, 37. nonanol, 38. naphtalène, 39. 2-phényléthanol, 40. m/e 82.39.64.112, 41. méthylcétone, 42. $\beta$-phényl éthyl acétate, 43. benzothiazole, 44. 2-undécénone, 45. 2-undécanone, 46. tridécane, 47. undécanol, 48. méthyl naphtalène, 49. méthylcétone, 50. méthylcétone, 51. éthyl décanoate, 52. 2-dodécanone, 53. diméthylnaphtalène, 54. hydrocarbure, 55. 2-tridécénone, 56. 2-tridécanone, 57. indole, 58. pentadécane. 
TABLEAU 6

Indice d'acidité de la matière grasse $(\mathrm{ml} \mathrm{NaOH} 0,01 \mathrm{~N}$ par g)

\begin{tabular}{ll|ll|ll}
\hline $2 \mathrm{j}$ & 5,5 & $6 \mathrm{j}$ & 6,5 & $9 \mathrm{j}$ & 9,8 \\
\hline
\end{tabular}

TABLEAU 7

Acides gras libres ( $\mu$ eq ac. palmitique pour $5 \mathrm{~g}$ de fromage)

\begin{tabular}{ll|ll|ll}
\hline $2 \mathrm{j}$ & 1260 & $6 \mathrm{j}$ & 1200 & $9 \mathrm{j}$ & 500 \\
\hline
\end{tabular}

\section{DISCUSSION}

Les travaux décrivant l'action lipolytique des psychrotrophes sont nombreux aussi n'est-il pas étonnant de constater en premier lieu l'apparition d'un goût de rance qui va de pair avec une augmentation de l'acidité de la matière grasse. Plus originale est la très forte augmentation des teneurs en méthylcétones qui n'avaient jamais été rapportées jusqu'alors. Pourtant cette augmentation n'est pas surprenante puisque ces méthylcétones sont formées à partir des acides gras libres libérés par les lipases ce qui explique que l'on constate une baisse de teneur en acides gras libres à longue chaîne dans les échantillons à forte teneur en composés carbonylés. Ces méthylcétones sont à leur tour réduites en alcools secondaires, et il est bien probable que les défauts de goûts constatés sont dus à la conjonction des acides gras libres, des méthylcétones et des alcools secondaires d'autant que ces derniers étant à longue chaîne $\left(\mathrm{C}_{\mathrm{i}} \mathrm{à} \mathrm{C}_{11}\right)$ ils ont des seuils de perception de même ordre de grandeur que celui des méthylcétones.

On peut en outre remarquer que dans les échantillons où la matière grasse est la plus dégradée on voit apparaître des cétones insaturées en quantités très supérieures à la normale (2-nonénone) et même des méthylcétones insaturées qui n'avaient jusqu'alors été isolées (2-tridécénone). Cette remarque vaut également pour les méthylcétones à nombre pair d'atomes de carbone qui apparaissent ici à des teneurs dépassant même celles de fromages bien affinés fabriqués au lait cru.

En ce qui concerne les esters on ne note aucune différence entre les échantillons alors que l'on aurait pu s'attendre à voir apparaître des esters éthyliques dans les fromages fabriqués avec des laits à forte population de psychrotrophes compte tenu des observations de 
Hosono et al. (1974) sur l'aptitude de ces micro-organismes à produire ces esters.

L'absence de goût ammoniacal remarqué à la dégustation, la texture, la faible quantité de produits soufrés, en particulier l'absence de 2,4-dithiapentane, et enfin, le fait que les produits volatils résultant de la dégradation des protéines ne soient pas plus abondants dans les échantillons issus de laits conservés le plus longtemps au froid confirment l'observation faite sur la coupe du fromage et qui montre que ces fromages s'affinent mal. On peut tout d'abord invoquer l'absence de flore bactérienne protéolytique de surface résultant de la technologie utilisée mais aussi le rôle inhibiteur des acides gras libres sur la croissance des bactéries normalement responsables de la protéolyse. Ceci n'est pas incompatible avec l'activité protéolytique des bactéries psychrotrophes observée par divers auteurs (Kjuru et al., 1971 ; Feuillet et al., 1976). Il ne serait pas impossible en effet que ces bactéries ne dégradent les protéines que jusqu'à un stade intermédiaire étant ainsi responsables de l'apparition de produits amers. Ces derniers ne sont pas ultérieurement dégradés du fait de l'inhibition des bactéries lactiques. Notons enfin que Law et al. (1976) travaillant, il est vrai, sur le Cheddar, restent muets quant au ralentissement de la protéolyse dans des fromages où ils observent un rancissement important dû aux psychrotrophes.

Notons enfin que seul l'échantillon fabriqué à partir de lait peu contaminé contient de l'acétoïne ce qui traduit le caractère réducteur des psychrotrophes.

On peut remarquer qu'il n'y a pas de variation dans les teneurs de deux métabolites très caractéristiques des pâtes molles le 1-3diméthoxybenzène et l'octen-1-ol-3 ce qui peut s'expliquer, pour ce dernier du moins, par le fait qu'il résulte de l'activité du Penicillium qui joue un rôle principal dans l'affinage des pâtes molles de lait pasteurisé.

Tous ces résultats dont certains sont nouveaux sur le plan analytique ne font, au fond, que confirmer une fois encore qu'il existe une relation étroite entre la bonne ou la mauvaise qualité bactériologique du lait et la bonne ou mauvaise qualité des produits préparés à partir de ce lait. De plus ils montrent que les phénomènes biochimiques responsables de défauts graves peuvent apparaître avant même que la population psychrotrophe n'ait dépassé le seuil parfois considéré comme critique de 1 million de germes.

\section{Résumé}

On a étudié l'influence de la contamination du lait de fabrication par des bactéries psychrotrophes sur les qualités organoleptiques de 
fromages à pâte molle. En plus d'une lipolyse accentuée, on a constaté une très forte augmentation des teneurs en méthylcétones et en alcools secondaires. La dégradation des protéines semble s'arrêter à un stade intermédiaire comme en témoigne l'amertume observée.

\section{S u m m a r y}

The effect of psychrotrophs in stored milk on the development of off flavour in soft cheese has been studied. An increase of volatile fatty acid but also of 2-alkanones and 2-alkanols has been observed. The levels of octen-1-ol-3 and 1-3-dimethoxybenzene are not modified. Bitterness increases also in the experimental cheeses.

Reçu pour publication en août 1977.

\section{Références}

Dumont (J. P.), Roger (Sylviane) et Adda (J.) (1974). - Le Lait, 54, 531-532.

Feuillet (M.), Guennec (S. LE) et Olsson (A.) (1976). - Le Lait, 56, 521-536.

Hosono (A.), Elliot (J. A.) and Mc Gugan (W. A.) (1974). - J. Dairy Sci., 57 (5), 535-539.

KJuru (K.), Eklund (E.), Gyllenberg (H.) und Antila (M.) (1971). - Milchwissenschaft, 26 (3), 138-141.

Lauwerys (R. R.) (1969). - Anal. Biochem., 32, 331.

Law (B. A.), Sharpe (M. L.) and Chapman (H. R.) (1976). - J. Dairy Res., 43 (3), $459-468$.

Qvist (I. H.) and Von Sydow (E.) (1974). - J. Agr. Food Chem., 22, 1077.

Schwartz (D. P.), Haller (H. S.) and Keeney. (M.) (1963). - Anal. Chem., 35, 2191.

SchwartZ (D. P.) and Parks (O. W.) (1963). - J. Dairy Sci., 46, 989.

Von Sydow (E.), Anderson (J.), Anjou (K.), Karlsson (Gunilla), Land (D.) and GRIFFIths (N.) (1970). - Lebensm. Wiss. u. Technol., 3, 11. 